Weizhou Wang*

\title{
Chalcogen bonds in the crystal structure of 4,7-dibromo-2,1,3-benzoselenadiazole, $\mathrm{C}_{6} \mathrm{H}_{2} \mathrm{Br}_{2} \mathrm{~N}_{2} \mathrm{Se}$
}
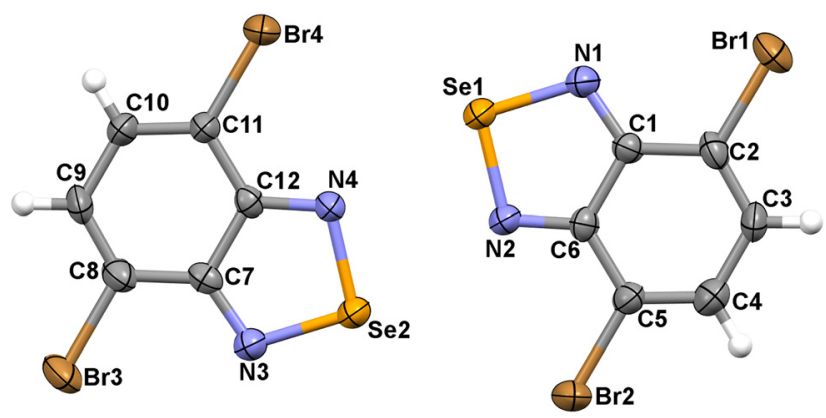

Figure 1: Molecular structure of the title compound with displacement ellipsoids draw at the $50 \%$ probability level.

https://doi.org/10.1515/ncrs-2021-0425

Received October 29, 2021; accepted December 2, 2021. published online December 14, 2021

\section{Abstract}

$\mathrm{C}_{6} \mathrm{H}_{2} \mathrm{Br}_{2} \mathrm{~N}_{2} \mathrm{Se}$, monoclinic, $P 2_{1} / c$ (no. 14), $a=7.7277(4) \AA$, $b=19.6360(6) \AA, c=10.8656(5) \AA, \beta=102.124(4)^{\circ}$, $V=1611.98(12) \AA^{3}, Z=4, R_{g t}(F)=0.0482, w R_{r e f}\left(F^{2}\right)=0.1175$, $T=290 \mathrm{~K}$.

\section{CCDC no.: 2125766}

The asymmetric unit of the title crystal structure is shown in the Figure 1. Table 1 contains crystallographic data and Table 2 contains the list of the atoms including atomic coordinates and displacement parameters.

\section{Source of material}

The 4,7-dibromo-2,1,3-benzoselenadiazole was purchased from Alfa Chemical Co. Ltd. (Zhengzhou, China) and used without further purification. The 4,7-dibromo-2,1,3-benzoselenadiazole (3.41 $\mathrm{mg}, 0.01 \mathrm{mmol}$ ) was dissolved in approximately $10 \mathrm{~mL}$ of methanol with gentle stirring at room temperature. Then, the solution was set aside for

*Corresponding author: Weizhou Wang, College of Chemistry and Chemical Engineering, Luoyang Normal University, Luoyang 471934, China, E-mail: wzw@lynu.edu.cn. https://orcid.org/0000-00024309-9077

Ә Open Access. @ 2021 Weizhou Wang, published by De Gruyter. (cc) BY License.
Table 1: Data collection and handling.

\begin{tabular}{ll}
\hline Crystal: & Brown block \\
Size: & $0.32 \times 0.28 \times 0.17 \mathrm{~mm}$ \\
Wavelength: & Mo K $\alpha$ radiation $(0.71073 \AA)$ \\
$\mu:$ & $14.5 \mathrm{~mm}^{-1}$ \\
Diffractometer, scan mode: & SuperNova, $\omega$ \\
$\theta_{\text {max }}$, completeness: & $28.5^{\circ},>99 \%$ \\
$N\left(h k l_{\text {measured }}, N(h k l)_{\text {unique }}, R_{\text {int }}:\right.$ & $18,469,3636,0.075$ \\
Criterion for $I_{\text {obs }}, N(h k l)_{\mathrm{gt}}:$ & $I_{\text {obs }}>2 \sigma\left(I_{\text {obs }}\right), 2375$ \\
$N(\text { param })_{\text {refined }}:$ & 199 \\
Programs: & CrysAlis \\
& SHELX [3] $[3]$, Olex2 [2], \\
\hline
\end{tabular}

crystallization by slow evaporation of the solvent at room temperature conditions. After about two days, brown block crystals of title compound suitable for single-crystal X-ray diffraction were obtained.

\section{Experimental details}

The structure was solved with the SHELXT [3] program using Intrinsic Phasing and refined with the SHELXL [4] refinement package. $\mathrm{H}$ atoms were placed in calculated positions and were included in the refinement in the riding model approximation, with $U_{\text {iso }}(\mathrm{H})$ set to $1.2 U_{\text {eq }}(\mathrm{C})$.

\section{Comment}

The term chalcogen bond was coined in 2009 [5]. Ten years later, the International Union of Pure and Applied Chemistry (IUPAC) issued a formal definition of the chalcogen bond [6]. In the crystals involving 2,1,3-benzoselenadiazole or its derivatives, the four-membered $[\mathrm{Se}-\mathrm{N}]_{2}$ cyclic double chalcogen bonds were always formed [7]. Besides the cyclic double chalcogen bonds, the other types of chalcogen bonds can also be formed between the 2,1,3-benzoselenadiazole or its derivatives and their coformers [8]. In this work, we studied the non-covalent interactions in the crystal of 4,7-dibromo2,1,3-benzoselenadiazole. The focus of attention is: what kind of chalcogen bond can be formed in the title crystal. 
Table 2: Fractional atomic coordinates and isotropic or equivalent isotropic displacement parameters $\left(\AA^{2}\right)$.

\begin{tabular}{lrrrr}
\hline Atom & $\boldsymbol{x}$ & $\boldsymbol{y}$ & $\boldsymbol{z}$ & $\boldsymbol{U}_{\text {iso }} \boldsymbol{U}_{\text {eq }}$ \\
\hline Br1 & $0.51340(11)$ & $0.82695(4)$ & $0.67016(8)$ & $0.0569(2)$ \\
Br2 & $0.56356(10)$ & $0.50297(4)$ & $0.82913(7)$ & $0.0510(2)$ \\
Se1 & $0.25275(9)$ & $0.61923(3)$ & $0.44783(6)$ & $0.0386(2)$ \\
N1 & $0.3403(7)$ & $0.6984(3)$ & $0.5138(5)$ & $0.0380(13)$ \\
N2 & $0.3542(6)$ & $0.5706(3)$ & $0.5841(5)$ & $0.0330(12)$ \\
C1 & $0.4348(8)$ & $0.6850(3)$ & $0.6276(6)$ & $0.0301(14)$ \\
C2 & $0.5266(8)$ & $0.7341(3)$ & $0.7143(6)$ & $0.0377(16)$ \\
C3 & $0.6242(8)$ & $0.7143(3)$ & $0.8267(6)$ & $0.0418(17)$ \\
H3 & 0.6848 & 0.7469 & 0.8815 & $0.050^{*}$ \\
C4 & $0.6360(9)$ & $0.6440(4)$ & $0.8628(6)$ & $0.0437(17)$ \\
H4 & 0.7041 & 0.6315 & 0.9406 & $0.052^{*}$ \\
C5 & $0.5482(8)$ & $0.5952(3)$ & $0.7843(6)$ & $0.0338(15)$ \\
C6 & $0.4431(8)$ & $0.6140(3)$ & $0.6654(5)$ & $0.0299(14)$ \\
Br3 & $-0.01261(10)$ & $0.22100(4)$ & $0.31893(8)$ & $0.0592(3)$ \\
Br4 & $-0.06430(10)$ & $0.54466(4)$ & $0.15761(7)$ & $0.0482(2)$ \\
Se2 & $0.25383(8)$ & $0.42750(4)$ & $0.53765(6)$ & $0.0379(2)$ \\
N3 & $0.1699(7)$ & $0.3480(3)$ & $0.4694(5)$ & $0.0367(13)$ \\
N4 & $0.1468(6)$ & $0.4774(2)$ & $0.4045(5)$ & $0.0340(12)$ \\
C7 & $0.0702(8)$ & $0.3615(3)$ & $0.3575(6)$ & $0.0306(14)$ \\
C8 & $-0.0253(8)$ & $0.3134(3)$ & $0.2715(6)$ & $0.0399(16)$ \\
C9 & $-0.1235(9)$ & $0.3331(3)$ & $0.1608(6)$ & $0.0410(17)$ \\
H9 & -0.1855 & 0.3008 & 0.1063 & $0.049^{*}$ \\
C10 & $-0.1345(8)$ & $0.4035(3)$ & $0.1250(6)$ & $0.0377(16)$ \\
H10 & -0.2027 & 0.4159 & 0.0471 & $0.045^{*}$ \\
C11 & $-0.0476(8)$ & $0.4523(3)$ & $0.2019(6)$ & $0.0322(14)$ \\
C12 & $0.0582(7)$ & $0.4333(3)$ & $0.3212(5)$ & $0.0269(13)$ \\
\hline & & & &
\end{tabular}

All bond lengths and angles in the title crystal structure are in the normal ranges. The 4,7-dibromo-2,1,3-benzoselenadiazole molecules form 2D corrugated sheets which further self-assemble into a 3D layered structure. There are five types of non-covalent interactions in the crystal structure: the four-membered [Se-N] $]_{2}$ cyclic double chalcogen bonds, $\mathrm{N}-\mathrm{Se} \cdots \mathrm{Br}$ chalcogen bonds, $\mathrm{C}-\mathrm{H} \cdots \mathrm{N}$ hydrogen bonds, $\pi \cdots \pi$ stacking interactions and $\sigma$-hole $(\mathrm{Br}) \cdots \sigma$-hole $(\mathrm{Br})$ stacking interactions $[9,10]$; among them, the four-membered $[\mathrm{Se}-\mathrm{N}]_{2}$ cyclic double chalcogen bonds and $\pi \cdots \pi$ stacking interactions are much stronger. According to the dispersioncorrected density functional theory calculations at the PBE0-D3/def2-TZVPP level of theory [11, 12], the binding energy of the four-membered [Se- $\mathrm{N}]_{2}$ cyclic double chalcogen bonds between two 4,7-dibromo-2,1,3-benzoselenadiazole molecules is $10.27 \mathrm{kcal} / \mathrm{mol}$, and the binding energy of the $\pi \cdots \pi$ stacking interactions between two 4,7-dibromo2,1,3-benzoselenadiazole molecules is $8.55 \mathrm{kcal} / \mathrm{mol}$. The details and reliability of the PBE0-D3/def2-TZVPP calculations for the study of the non-covalent interactions can be found elsewhere [13-15]. Evidently, the larger binding energy of the four-membered $[\mathrm{Se}-\mathrm{N}]_{2}$ cyclic double chalcogen bonds between two 4,7-dibromo-2,1,3-benzoselenadiazole molecules explains why the four-membered $[\mathrm{Se}-\mathrm{N}]_{2}$ cyclic double chalcogen bonds were always found in the crystals involving 2,1,3-benzoselenadiazole or its derivatives.

In conclusion, there are two types of chalcogen bonds in the crystal of 4,7-dibromo-2,1,3-benzoselenadiazole. One type is of the much stronger four-membered $[\mathrm{Se}-\mathrm{N}]_{2}$ cyclic double chalcogen bonds, and the other is of the much weaker $\mathrm{N}-\mathrm{Se} \cdots \mathrm{Br}$ chalcogen bonds.

Acknowledgment: Computer time was provided by the National Supercomputing Center in Shenzhen.

Author contributions: The author has accepted responsibility for the entire content of this submitted manuscript and approved submission.

Research funding: This work was supported by the National Science Foundation of China (Grant No. 21773104) and the Program for Science \& Technology Innovation Talents in Universities of Henan Province (Grant No. 13HASTIT015).

Conflict of interest statement: The author declares no conflicts of interest regarding this article.

\section{References}

1. Rigaku. CRYSALIS ${ }^{\mathrm{Pro}}$; Rigaku Inc: Tokyo, Japan, 2015.

2. Dolomanov O. V., Bourhis L. J., Gildea R. J., Howard J. A. K., Puschmann H. OLEX2: a complete structure solution, refinement and analysis program. J. Appl. Crystallogr. 2009 , 42, 339-341.

3. Sheldrick G. M. SHELXTL - integrated space-group and crystalstructure determination. Acta Crystallogr. 2015, A71, 3-8.

4. Sheldrick G. M. Crystal structure refinement with SHELXL. Acta Crystallogr. 2015, C71, 3-8.

5. Wang W., Ji B., Zhang Y. Chalcogen bond: a sister noncovalent bond to halogen bond. J. Phys. Chem. 2009, 113, 8132-8135.

6. Aakeroy C. B., Bryce D. L., Desiraju G. R., Frontera A., Legon A. C., Nicotra F., Rissanen K., Scheiner S., Terraneo G., Metrangolo P., Resnati G. Definition of the chalcogen bond. Pure Appl. Chem. 2019, 91, 1889-1892.

7. Eichstaedt K., Wasilewska A., Wicher B., Gdaniec M., Połoński T. Supramolecular synthesis based on a combination of Se...N secondary bonding interactions with hydrogen and halogen bonds. Cryst. Growth Des. 2016, 16, 1282-1293.

8. Miao S., Zhang Y., Shan L., Xu M., Wang J. G., Zhang Y., Wang W. A robust supramolecular heterosynthon assembled by a hydrogen bond and a chalcogen bond. Crystals 2021, 11, 1309.

9. Zhang Y., Wang W. The $\sigma$-hole... $\sigma$-hole stacking interaction: an unrecognized type of noncovalent interaction. J. Chem. Phys. 2020, 153, 214302.

10. Zhang Y., Wang W. The face-to-face $\sigma$-hole... $\sigma$-hole stacking interactions: structures, energies, and nature. Crystals 2021, 11, 877.

11. Adamo C., Barone V. Toward reliable density functional methods without adjustable parameters: the PBE0 model. J. Chem. Phys. $1999,110,6158-6169$. 
12. Grimme S., Antony J., Ehrlich S., Krieg H. A consistent and accurate $a b$ initio parametrization of density functional dispersion correction (DFT-D) for the 94 elements H-Pu. J. Chem. Phys. 2010, 132, 154104.

13. Wang W., Zhang Y., Wang Y. B. Noncovalent $\pi . . . \pi$ interaction between graphene and aromatic molecule: structure, energy, and nature. J. Chem. Phys. 2014, 140, 094302.
14. Wang W., Sun T., Zhang Y., Wang Y. B. The benzene...naphthalene complex: a more challenging system than the benzene dimer for newly developed computational methods. J. Chem. Phys. 2015, 143, 114312.

15. Wang W., Zhang Y., Wang Y. B. Highly accurate benchmark calculations of the interaction energies in the complexes $\mathrm{C}_{6} \mathrm{H}_{6} \ldots$ $\mathrm{C}_{6} \mathrm{X}_{6}(\mathrm{X}=\mathrm{F}, \mathrm{Cl}, \mathrm{Br}$, and I). Int. J. Quant. Chem. 2017, 117, e25345. 